\title{
A NOTE ON STRASSEN'S VERSION OF THE LAW OF THE ITERATED LOGARITHM
}

\author{
A. K. BASU
}

Abstract. Strassen's law of the iterated logarithm is extended to stationary ergodic martingales and to a non-identically-distributed case.

1. Introduction. V. Strassen [6] proved that if $\left\{x_{n}\right\}$ are i.i.d.r.v.'s with $E x=0, E x^{2}=1$, then for a continuous map $\phi$ from $C[0,1]$ to $R^{1}$, the sequence $\phi\left(\eta_{n}\right)$ is relatively compact and the set of its limit points coincides with $\phi(K)$ where $\eta_{n}$ is obtained by linearly interpolating

$$
\begin{aligned}
(2 n \log \log n)^{-1 / 2} S_{i} & \text { at } t=i / n, i=1,2, \cdots, n, \\
& \text { and } S_{i}=x_{1}+\cdots+x_{i} .
\end{aligned}
$$

$K$ is the set of absolutely continuous functions $f \in C[0,1]$ such that $f(0)=0$ and $\int_{0}^{1}\left|f^{\prime}(t)\right|^{2} d t \leqq 1$.

The key idea of the paper is that Strassen's theorem holds for any random sequence for which there is a Skorohod embedding in Brownian motion with stopping times $\tau_{n}$ satisfying the strong law of large numbers. Two examples are given: one is the stationary ergodic martingales and the other a non-identically-distributed case.

As an easy extension of the corollary of Strassen [6], we can obtain in particular if $\left\{x_{i}, F_{i}, i \geqq 1\right\}$ is a stationary ergodic stochastic sequence $E\left(x_{i} \mid F_{i-1}\right)=0$ a.s. for all $i \geqq 1$ and $E x_{1}^{2}=1$, then

$$
\lim \sup S_{n}(2 n \log \log n)^{-1 / 2}=1 \text { a.s. }
$$

and

$$
\lim \sup \frac{1}{n_{i=1}} \sum_{i=1}^{n} S_{i} /\left(\frac{2}{3} n \log \log n\right)^{1 / 2}=1 \text { a.s. }
$$

Received by the editors March 23, 1970 and, in revised form, January. 19, 1971 and November 17, 1972.

AMS (MOS) subject classifications (1970). Primary 60F15, 60G50; Secondary 60F05.

Key words and phrases. Iterated logarithm, Brownian motion, martingale difference, representation theorem, stationary ergodic.

(c) American Mathematical Society 1973 
(1) is recently obtained by W. F. Stout [7] by a different method and (2) is an extension of the iterated logarithm for "Cesàro sums" considered by Gal and Stackelberg [5], and Gaposhkin [2].

\section{Results.}

LEMMA 1. If $\left\{\tau_{i}\right\}$, the sequence of stopping times of the Skorohod representation theorem, satisfies the strong law of large numbers namely

$$
\left(\tau_{1}+\cdots+\tau_{k}\right) / k \rightarrow 1 \text { a.s. }
$$

then there is a probability space with a Brownian motion $B(t)$ and a sequence $\left\{\widetilde{S}_{n}\right\}_{1}^{\infty}$ having the same distribution as $\left\{S_{n}\right\}_{1}^{\infty}$ such that

$$
\sup _{\tau \leqq t} \frac{\left|\tilde{S}_{\tau}-B(\tau)\right|}{(2 t \log \log t)^{1 / 2}} \rightarrow 0 \quad \text { a.s. as } t \rightarrow \infty .
$$

Proof. Follows from [6].

THEOREM 1. Strassen's theorem [6] holds for a stationary ergodic martingale difference sequence $\left\{x_{n}, F_{n}, n \geqq 1\right\}$ with $E x_{1}^{2}=1$.

To facilitate the proof of Theorems 1 and 2 let us state some known results from Jonas's thesis [3] and often we shall sketch a proof.

A generalization of Skorohod's theorem. Let (A) $\hat{X}_{1}, \hat{X}_{2}, \cdots$ be a sequence of random variables on a probability space $(\hat{\Omega}, \hat{B}, \hat{P})$ for which $E\left(\hat{X}_{n}^{2} \mid \hat{X}_{1}, \cdots, \hat{X}_{n}\right)$ exists for all $n$ and $E\left(\hat{X}_{n} \mid \hat{X}_{1}, \cdots, \hat{X}_{n}\right)=0$ a.s.

Let $(\Omega, \mathscr{B}, P)$ be a probability space with the following properties:

(1) There exists a Brownian motion $B=B(t, \omega)$ on $(\Omega, \mathscr{B}, P)$.

(2) There exist random variables independent of the Brownian motion $B, Y_{1}, Y_{2}, \cdots$ on $(\Omega, \mathscr{B}, P)$ which are pairwise independent and distributed uniformly over the interval $[0,1]$.

Then there exists a sequence of nonnegative random variables $\tau_{1}, \tau_{2}, \cdots$ on $(\Omega, \mathscr{B}, P)$ and $(\mathrm{B})$ random variables $X_{1}, X_{2}, \cdots$ on $(\Omega, \mathscr{B}, P)$, such that r.v.'s of the sequence (A) and (B) have the same distribution and $\sum_{1}^{n} X_{i}=B\left(\sum_{1}^{n} \tau_{i}\right)$ a.s.

Moreover, if $\mathscr{A}_{n}=\mathscr{B}\left(X_{1}, X_{2}, \cdots, X_{n}, B(t), 0 \leqq t \leqq \sum_{j=1}^{n} \tau_{j}\right)$, i.e. the $\sigma$-field generated by $X_{1}, \cdots, X_{n}$ and $B(t)\left(0 \leqq t \leqq \sum_{1}^{n} \tau_{j}\right)$, then the following hold.

(1) $\tau_{n}$ is $\mathscr{A}_{n}$-measurable.

(2) For each $s>0, B_{n}(s)=B\left(\sum_{j=1}^{n} \tau_{j}+s\right)-B\left(\sum_{j=1}^{n} \tau_{j}\right)$ is independent of $\mathscr{A}_{n}$.

(3) $E\left(\tau_{n} \mid \mathscr{A}_{n-1}\right)$ exists and $E\left(\tau_{n} \mid \mathscr{A}_{n-1}\right)=E\left(X_{n}^{2} \mid \mathscr{A}_{n-1}\right)$ a.s. $=E\left(X_{n}^{2} \mid X_{1}\right.$, $\left.X_{2}, \cdots, X_{n-1}\right)$. 
(4) If $k>0$ and if $E\left(X_{n}^{2 k} \mid X_{1}, \cdots, X_{n-1}\right)$ exists, then $E\left(\tau_{n}^{k} \mid \mathscr{A}_{n-1}\right)$ exists too, and further

$$
E\left(\tau_{n}^{k} \mid \mathscr{A}_{n-1}\right) \leqq L_{k} E\left(X_{n}^{2 k} \mid \mathscr{A}_{n-1}\right)=L_{k} E\left(X_{n}^{2 k} \mid X_{1}, \cdots, X_{n-1}\right) \quad \text { a.s. }
$$

where $L_{k}$ is a constant depending only on $k$.

SKETCH OF PROOF. Like Skorohod [4, pp. 163-164], let us state

Lemma 2. Suppose that $B(t)$ is a Brownian motion process for $t \geqq 0$ and $B(0)=0$ and $\tau_{n+1}$ is the smallest root of $\left(B_{n}(t)-a\right)\left(B_{n}(t)-b\right)=0$, where $a<0<b$. Then for every $\lambda>0$,

$$
\left.E\left(\exp \left(-\lambda \tau_{n+1}\right) I_{\left[B_{n}\left(\tau_{n+1}\right)=b\right]} \mid \mathscr{A}_{n}\right)=\sinh b(2 \lambda)^{1 / 2} / \sinh , b-a\right)(2 \lambda)^{1 / 2} \text { a.s. }
$$

and

$E\left(\exp \left(-\lambda \tau_{n+1}\right) I_{\left[B_{n}\left(\tau_{n+1}\right)=b\right]} \mid \mathscr{A}_{n}\right)=-\sinh a(2 \lambda)^{1 / 2} / \sinh (b-a)(2 \lambda)^{1 / 2} \quad$ a.s.

We can prove the lemma and the theorem by induction i.e. we would assume that $\tau_{1}, \tau_{2}, \cdots, \tau_{n}$ and $X_{1}, X_{2}, \cdots, X_{n}$ are already constructed having the desired properties. We now consider the construction of $\tau_{n+1}$.

Let $f_{a}=\exp \left(-\lambda \tau_{n+1}\right) I_{\left[B_{n}\left(\tau_{n+1}\right)=a\right]}$. By assumption $B_{n}(t)$ is independent of $\mathscr{A}_{n}$ and, by definition, of $\tau_{n+1} . \mathscr{B}\left(f_{a}\right) \subset \mathscr{B}\left(B_{n}(t), t \geqq 0\right)$; therefore $\mathscr{B}\left(f_{a}\right)$ is independent of $\tau_{n}$. Like Skorohod [4, p. 166] let us state a

COROLlaRY. If $B_{n}(t), \tau_{n+1}$ is as above, then for $\lambda>0$ the following hold:

$$
\begin{aligned}
E\left(\exp \left(-\lambda \tau_{n+1}\right) \mid \mathscr{A}_{n}\right) & =\frac{\sinh b(2 \lambda)^{1 / 2}-\sinh a(2 \lambda)^{1 / 2}}{\sinh (b-a)(2 \lambda)^{1 / 2}} \text { a.s.; } \\
E\left(I_{\left[B_{n}\left(\tau_{n+1}\right)=a\right]} \mid \mathscr{A}_{n}\right) & =b /(b-a) \text { and } \\
E\left(I_{\left[B_{n}\left(\tau_{n+1}\right)=b\right]} \mid \mathscr{A}_{n}\right) & =-a /(b-a) ; \\
E\left(\tau_{n+1} \mid \mathscr{A}_{n}\right) & =-a b \quad \text { a.s }
\end{aligned}
$$

(4) For each $k>0$, there exists a constant $C_{k}$ depending on $k$ only such that $E\left(\tau_{n+1}^{k} \mid \mathscr{A}_{n}\right) \leqq C_{k} a b(b-a)^{2 k-2}$.

Here also the $\sigma$-field generated by those r.v.'s whose conditional expectation will be constructed is contained in the $\sigma$-field $\mathscr{B}\left(B_{n}(t), t \geqq 0\right)$ and hence independent of $\mathscr{A}_{n}$.

Let $F_{n}(x, u)$ be the conditional distribution function of $\hat{X}_{n+1}$ given $\left(X_{1}, X_{2}, \cdots, X_{n}\right)=x$. If the jumps (or discontinuities) of $F$ are joined by vertical lines and the graph so obtained is reversed then we obtain a function $f_{n}(x, t)$ on $R^{n} \times[0,1]$ to $R$. For fixed $x$ this $f_{n}(x, t)$, except for at most countable points $t$, is defined everywhere in $[0,1]$. These points are precisely those $t$ 's for which $F_{n}(x, u)=t . f_{n}(x, t)$ is the distribution 
function mapping. Since $E\left(\widehat{X}_{n+1} \mid \widehat{X}_{1}, \cdots, \widehat{X}_{n}\right)=0$, like Skorohod, we get $\int_{\alpha_{n}(x)}^{1} f_{n}(x, t) d t=-\int_{0}^{\alpha_{n}(x)} f_{n}(x, t) d t$ where

$$
\alpha_{n}(x)=F_{n}(x, 0)+\frac{1}{2}\left(\lim _{z \downarrow 0} F_{n}(x, z)-\lim _{z^{\dagger} 0} F_{n}(x, z)\right)
$$

and a function $G_{n}: R^{n} \times[0,1] \rightarrow[0,1]$ defined by

and

$$
\int_{\alpha_{n}(x)}^{\nu} f_{n}(x, t) d t=-\int_{G_{n}(x, y)}^{\alpha_{n}(x)} f_{n}(x, t) d t \quad\left(\text { if } 1 \geqq y \geqq \alpha_{n}(x)\right)
$$

$$
\left.\int_{x}^{\alpha_{n}(x)} f_{n}(x, t) d t=-\int_{\alpha_{n}(x)}^{G_{n}(x, y)} f_{n}(x, t) d t \quad \text { (if } 0 \leqq y \leqq \alpha_{n}(x)\right)
$$

and also $G_{n}$ has the property

$$
G_{n}\left(x, G_{n}(x, y)\right)=x \quad \text { a.s. }(y \in[0,1]), \quad G_{n}\left(x, \alpha_{n}(x)\right)=\alpha_{n}(x) \text { a.s. }
$$

Let $Y_{n+1}$ be the $(n+1)$ th uniformly distributed random variable on $(\Omega, \mathscr{B}, P)$. Then we can define two mappings $X_{n+1}^{a}$ and $X_{n+1}^{b}$ from $R^{n} \times$ $[0,1]$ to $R$ by

$$
X_{n+1}^{a}(x, \omega)=f_{n}\left(x, Y_{n+1}(\omega)\right), \quad X_{n+1}^{b}(x, \omega)=f_{n}\left(x, G_{n}\left(x, Y_{n+1}(\omega)\right) .\right.
$$

$X_{n+1}^{a}$ and $X_{n+1}^{b}$ are $\mathscr{B}^{n} \times \mathscr{B}\left(Y_{n+1}\right)$ measurable random variables where $\mathscr{B}^{n}$ is the usual Borel field on $R^{n}$ and $\mathscr{B}\left(Y_{n+1}\right)$ is the Borel field defined by $Y_{n+1}$. Like Skorohod [4, p. 167] we state the lemma.

LEMMA 3. Let $B_{n}(t)$ be independent of $\mathscr{A}_{n}$ and $Y_{n+1}$. Let $T(x, \eta)$, $x \in R^{n}, \eta \in[0,1]$ be the smallest solution of

$$
\left(B_{n}(t, \eta)-X_{n+1}^{a}(x, \eta)\right)\left(B_{n}(t, \eta)-X_{n+1}^{b}(x, \eta)\right)=0 .
$$

Further let $T_{n+1}(\omega)=T\left(X_{1}(\omega), \cdots, X_{n}(\omega), \omega\right)$ and $X_{n+1}=B_{n}\left(T_{n+1}\right)$, then $T_{n+1}$ is $\mathscr{A}_{n+1}$ measurable and $X_{1}, X_{2}, \cdots, X_{n+1}$, and $\hat{X}_{1}, \hat{X}_{2}, \cdots, \hat{X}_{n+1}$ have the same distribution.

Let $F_{i}$ be the $\sigma$-field generated by $X_{1}, X_{2}, \cdots, X_{i}$ and $F_{0}=\mathscr{A}_{0}=\{\phi, \Omega\}$.

Proof of Theorem 1. Now by the Birkhoff ergodic theorem

$$
\begin{aligned}
n^{-1}\left[E\left(\tau_{n} \mid \mathscr{A}_{n-1}\right)+E\left(\tau_{n-1} \mid \mathscr{A}_{n-2}\right)\right. & \left.+\cdots+E\left(\tau_{2} \mid \mathscr{A}_{1}\right)+E\left(\tau_{1} \mid \mathscr{A}_{0}\right)\right] \\
& \rightarrow \frac{1}{n} \sum_{i=1}^{n} E\left(X_{i}^{2} \mid F_{i-1}\right) \rightarrow E X_{1}^{2}=1 \quad \text { a.s.; }
\end{aligned}
$$

now it is enough to show that

$$
\frac{1}{n} \sum_{i=1}^{n}\left[\tau_{i}-E\left(\tau_{i} \mid \mathscr{A}_{i-1}\right)\right] \rightarrow 0 \quad \text { a.s.; }
$$


using Theorem 5 of Chow [1], the result will follow if

Now

$$
\sum_{n=1}^{\infty} \frac{1}{n^{2}} E\left(\tau_{n}-E\left(\tau_{n} \mid \mathscr{A}_{n-1}\right)\right)^{2}<\infty
$$

$$
\sum_{n=1}^{\infty} \frac{1}{n^{2}} E\left(\tau_{n}-E\left(\tau_{n} \mid \mathscr{A}_{n-1}\right)\right)^{2} \leqq \sum_{n=1}^{\infty} \frac{1}{n^{2}} E \tau_{n}^{2} \leqq L_{2} \sum_{n=1}^{\infty} \frac{1}{n^{2}} E X_{n}^{4}
$$

Since $\left(X_{i}, i \geqq 1\right)$ is a stationary ergodic sequence with $E\left(X_{i} \mid F_{i-1}\right)=0$ a.s. for all $i \geqq 2$, proceeding as in Skorohod [4] we see that $\left(\tau_{i}, i \geqq 1\right)$ are stationary. Since $\left(Y_{n}, n \geqq 1\right)$ are independent identically distributed uniform random variables on $[0,1]$ and $\left(X_{n}, n \geqq 1\right)$ are stationary ergodic stochastic sequences, $\left\{X_{n}^{a}\right\}$ and $\left\{X_{n}^{b}\right\}$ are also stationary ergodic stochastic sequences.

Now by a truncated argument, e.g., considering $X_{n}^{*}=X_{n} I_{\left[\left|X_{n}\right|<\varepsilon \sqrt{ } n\right]}$, (6) is true. Therefore $n^{-1}\left(\tau_{1}+\tau_{2}+\cdots+\tau_{n}\right) \rightarrow 1$ a.s. So by Lemma 1 ,

$$
\sup _{\tau \leqq t} \frac{\left|\tilde{S}_{[\tau]}-B_{(\tau)}\right|}{(2 t \log \log t)^{1 / 2}} \rightarrow 0 \quad \text { a.s. as } t \rightarrow \infty .
$$

So the theorem follows from the Corollary to Theorem 3 of [6].

THEOREM 2. Strassen's theorem [6] holds if $x_{1}, x_{2}, \cdots$ are independent r.v.'s with $E x_{i}=0, E x_{i}^{2}=1$ and

$$
\sum_{k=2}^{\infty} \frac{E\left|x_{k}\right|^{2+\delta}}{k^{1+\delta / 2}}<\infty, \quad 2 \geqq \delta>0
$$

(in particular if $E\left|x_{k}\right|^{2+\delta} \leqq C$ for all $k$ ).

Proof. Now

$$
E\left|\tau_{k^{-1}}\right|^{1+\delta / 2} \leqq 2^{\delta}\left(E \tau_{k}^{1+\delta / 2}+1\right) \leqq 2^{\delta}\left(C_{\delta} E\left|x_{k}\right|^{2+\delta}+1\right) .
$$

So by Theorem 5 of Chow [1]

$$
\sum_{k=2}^{\infty} \frac{E\left|x_{k}\right|^{2+\delta}}{k^{1+\delta / 2}}<\infty \text { implies } \frac{1}{n} \sum_{1}^{n}\left(\tau_{i}-E \tau_{i}\right) \rightarrow 0 \text { a.s. }
$$

Therefore $\left\{\tau_{i}\right\}$ satisfies the conditions of Lemma 1 .

REMARKS 1. Gaposhkin [2] proved that if $x_{1}, x_{2}, \cdots$ are independent r.v.'s with $\left|x_{k}\right| \leqq C$ a.s. and $E x_{k}^{2}=1, E x_{k}=0$ for all $k=1,2, \cdots$ then

$$
\lim \sup \sum_{k=1}^{n}\left(1-\frac{k}{n}\right)^{\alpha} x_{k}\left(\frac{2}{2 \alpha+1} n \log \log n\right)^{-1 / 2}=1 \text { a.s., } \alpha>0 .
$$

We shall extend this result under the conditions of Theorems 1 and 2. 
Let $S_{0}=0$. By Abel's sum

$$
\begin{aligned}
\sum_{k=1}^{n}\left(1-\frac{k}{n}\right)^{\alpha} x_{k} & =\sum_{k=0}^{n-1}\left\{\left(1-\frac{k}{n}\right)^{\alpha}-\left(1-\frac{k+1}{n}\right)^{\alpha}\right\} S_{k} \\
& \cong \alpha \frac{1}{n} \sum_{k=0}^{n-1}\left(1-\frac{k}{n}\right)^{\alpha-1} S_{k} .
\end{aligned}
$$

Taking $f(t)=\alpha(1-t)^{\alpha-1}$ on p. 218 of Strassen [6] we get

$\limsup _{n \rightarrow \infty} \sum_{k=1}^{n}\left(1-\frac{k}{n}\right)^{\alpha} x_{k}(2 n \log \log n)^{1 / 2}$

$$
=\limsup _{n \rightarrow \infty}\left(2 n^{3} \log \log n\right)^{-1 / 2} \sum_{k=1}^{n} f\left(\frac{k}{n}\right) S_{k}=\left(\frac{1}{2 \alpha+1}\right)^{1 / 2} .
$$

Similarly Theorem 2 and Theorem 3 of Gaposhkin [2] may be extended.

2. We conjecture that if $x_{1}, x_{2}, \cdots$ is a martingale difference sequence with $E x_{1}=0, E x_{n}^{2}=1$ for all $n$ and $\left\{x_{n}^{2}\right\}_{1}^{\infty}$ is uniformly integrable, then the law of the iterated logarithm holds.

ACKNOWLEDgement. Appreciation is extended to the National Research Council of Canada for financial support of this work.

Thanks to the referee for some helpful suggestions.

\section{REFERENCES}

1. Y. S. Chow, Local convergence of martingales and the law of large numbers, Ann. Math. Statist. 36 (1965), 552-558. MR 31 \#6264.

2. V. F. Gapoškin, The law of the iterated logarithm for Cesaro's and Abel's method of summation, Teor. Verojatnost. i Primenen. 10 (1965), 449-459=Theor. Probability Appl. 10 (1965), 411-420. MR 33 \#3336.

3. F. Jonas, Unpublished Thesis, Erlangen, 1967.

4. A. V. Skorohod, Studies in the theory of random processes, Izdat. Kiev. Univ., Kiev, 1961 ; English transl., Addison-Wesley, Reading, Mass., 1965. MR 32 \#3082a,b.

5. O. Stackelberg, On the law of iterated logarithm. I, II, Nederl. Akad. Wetensch. Proc. Ser. A 67=Indag. Math. 26 (1964), 48-55, 56-67. MR 28 \#4558.

6. V. Strassen, Invariance principle for law of the iterated logarithm, Z. Wahrscheinlichkeitstheorie und Verw. Gebiete 3 (1964), 211-226. MR 30 \#5379.

7. W. F. Stout, The Hartman-Wintner law of the iterated logarithm for martingales, Ann. Math. Statist. 41 (1970), 2158-2160.

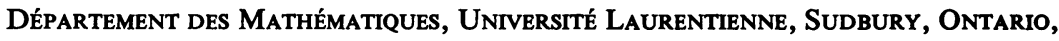
CANADA 